\title{
LOS JARALES DE LA CLASE CISTO-LAVANDULETEA EN EL SUR DE LA PENÍNSULA IBÉRICA (ANDALUCÍA, ESPAÑA)
}

\author{
Andrés V. PÉREZ LATORRE, Patricia NAVAS FERNÁNDEZ, \\ José María NIETO CALDERA y Baltasar CABEZUDO
}

\begin{abstract}
RESUMEN. Los jarales de la clase Cisto-Lavanduletea en el sur de la Península Ibérica (Andalucía, España). Se ha realizado una recopilación sobre las asociaciones de la clase fitosociológica CistoLavanduletea en el sur de la Península Ibérica (Andalucía, España). La gran riqueza fitocenológica viene avalada por la diversidad en Andalucía de ambientes requeridos por estas formaciones: suelos silíceos degradados, de textura compacta o arenosa, territorios desde el nivel del mar hasta cerca de los $1700 \mathrm{~m}$ y precipitaciones no muy escasas siempre con régimen pluviométrico y térmico de tipo mediterráneo. Las alianzas detectadas han sido Ulici argentei-Cistion ladaniferi (de suelos compactos y áreas térmicas lluviosas), Calicotomo spinosae-Cistion ladaniferi (de suelos compactos y áreas térmicas secas), Coremion albi (de suelos arenosos y áreas térmicas secas), Cistion laurifolii (de suelos compactos y áreas frías) y Stahelino-Ulicion baetici (sobre rocas ígneas ultrabásicas). Se han reconocido un total de 17 asociaciones, proponiéndose 1 nueva subalianza de areal aljíbico (Ulici borgiae-Calicotomenion villosae), 2 nuevas combinaciones (Genisto hirsutae-Cistetum ladaniferi cistetosum libanotis y Teucrio compacti-Cistetum ladaniferi cistetosum populifolii), I nueva subasociación (Lavandulo caesiae-Genistetum equisetiformis calicotometosum intermediae) y lectotipificándose una asociación (Teucrio mariani-Cistetum laurifolii).
\end{abstract}

Palabras clave. Vegetación preforestal mediterránea, jarales, Cisto-Lavanduletea, Andalucía, España.

RÉSUMÉ. Les cisteaies de la clase Cisto-Lavanduletea dans le Sud de la Péninsule Ibérique (Andalousie, Espagne). On a fait une récapitulation des références concernant la classe phytosociologique CistoLavanduletea dans le sud de la Péninsule Ibérique (Andalousie, Espagne). La grande richesse phytocénologique déconde de la diversité en Andalousie des environments requis pour ces formations: sols de silice dégradés, de texture compacte on sablonneuse, territoires deprés le niveau de la mer jusqu'a près de $1700 \mathrm{~m}$. et precipitations pas tiés faibles, toujours avec un régime pluviemetrique et thermique de type méditerranéen. Les alliances trouvées sont: Ulici argentei-Cistion ladaniferi (sols compacts et aires thermiques pluvieuses), Calicotomo spinosae-Cistion ladaniferi (de sols compacts et aires thermiques sèches), Coremion albi (de sols sabloneux et aires thermiques sèches), Cistion laurifolii (de sols compacts et aires froids) et Stahelino-Ulicion baetici (sur roches ignées ultrabasiques). On a reconnu, en tont, 17 associations, on propose une nouvelle sub-alliance d'une aire aljíbico (Ulici borgiae-Calicotomenion villosae), deux nouvelles combinaisons (Genisto hirsutae-Cistetum ladaniferi cistetosum libanotis et Teucrio compacti-Cistetum ladaniferi cistetosum populifolii), I nouvelle sub-association (Lavandulo caesiae-Genistetum equisetiformis calicotometosum intermediae) et on choisit un lectotypus pour 1 association (Teucrio mariani-Cistetum laurifolii).

Mots-clés. Végétation préforestière, cistes, Cisto-Lavanduletea, Andalousie, Espagne.

ABSTRACT. Mediterranean shrublands of Cisto-Lavanduletea in the South of the Iberian Peninsula (Andalusia, Spain). Shrublands silicicolous communities have been studied in the south of the Iberian 
Peninsula (Andalusia, Spain). They are included in the Cisto-Lavanduletea phytosociological class. The great diversity of this type of vegetation in Andalusia comes endorsed by the diverse environment required by these communities: siliceous demoted soils, with sandy or compact texture, territories from the sea level up to $1700 \mathrm{~m}$ and with rainfalls not very scarce, always under mediterranean seasonality. The recorded alliances have been the following: Ulici argentei-Cistion ladaniferi (on compact soils and rainy thermal areas), Calicotomo spinosae-Cistion ladaniferi (on compact soils and dry thermal areas), Coremion albi (on sandy soils and dry thermal areas), Cistion laurifolii (on compact soils and cold areas) and StahelinoUlicion baetici (on igneous peridotite rocks). An amount of 17 associations have been finally recognized. It is proposed 1 new suballiance in the Aljíbico sector (Ulici borgiae-Calicotomenion villosae), 2 new combinations (Genisto hirsutae-Cistetum ladaniferi cistetosum libanotis and Teucrio compacti-Cistetum ladaniferi cistetosum populifolii), 1 new subassociation (Lavandulo caesiae-Genistetum equisetiformis calicotometosum intermediae) and an association has been lectotypified (Teucrio mariani-Cistetum laurifolii).

Keywords. Pre-forest mediterranean vegetation, silicicolous shrublands, Cisto-Lavanduletea, Andalusia, Spain.

\section{INTRODUCCIÓN}

Los jarales son comunidades vegetales inçluíbles en las formaciones de matorral mediterráneo, heliófilas, xerófilas y silicícolas, que se instalan sobre suelos meso-oligotrofos y con óptimo en la región Mediterránea (Rivas Martínez et al., 1979; Molina Abril, 1993). Están caracterizados por la dominancia de especies del género Cistus y otras cistáceas, destacando por su grado de presencia: Cistus ladanifer, Cistus monspeliensis, Cistus laurifolius, Cistus salvifolius, Cistus populifolius, Halimium halimifolium, etc., generalmente acompañadas de una gran variedad de especies de los géneros Genista y Ulex.

Estas formaciones tienen gran importancia en la subregión Mediterránea Occidental (Rivas Martínez et al., op. cit.), concretamente en el sudoeste de la península Ibérica (Pérez Latorre et al., 1993), y el norte de Marruecos (Benabid et al., 1994). Constituyen etapas de sustitución de los bosques de Quercus tales como alcornocales (Quercus suber), encinares (Quercus rotundifolia) o robledales (Quercus pyrenaica) de áreas no muy lluviosas. Los jarales están ampliamente representados en Andalucía debido precisamente a la degradación sufrida históricamente por dichas formaciones (Galiano, 1990).

Son numerosos los datos que hacen referencia a los jarales en Andalucía aunque en general están dispersos en trabajos sobre otras unidades de vegetación. Las publicaciones que hacen mayor hincapié al respecto son las de Ceballos y Martín Bolaños (1930), Ceballos y Vicioso (1933), Rivas Goday (1964b), Rivas Goday y Rivas Martínez (1967), Rivas Martínez (1979), Martínez Parras et al. (1987), Quézel et al. (1988), Asensi y Díez Garretas (1988), Ladero et al. (1990), Cano et al. (1991), Peinado et al. (1992), Pérez Latorre et al. (1993) y Escudero et al. (1995). Datos sobre los jarales marroquíes (con proximidad fitogeográfica) aparecen en Benabid et al. (1994).

Los objetivos de este trabajo consisten en aportar los datos fitosociológicos, ecológicos y corológicos, a nivel de asociación, de los járales y jaguarzales comprendidos en la clase Cisto-Lavanduletea presentes en Andalucía, estudiándose además su status sintaxonómico.

\section{MATERIAL Y MÉTODOS}

\section{Área de estudio}

El territorio estudiado se circunscribe al 
sur de la Península Ibérica, concretamente a la Comunidad Autónoma de Andalucía (fig. 1). Biogeográficamente pertenece a la subregión Mediterránea Occidental e incluyendo en parte las siguientes provincias corológicas: TingitanoOnubo-Algarviense, Luso-Extremadurense, Bética (pertenecientes a la superprovincia Iberomarroquí-Atlántica, Pérez Latorre et al., 1996) y Murciano-Almeriense (superprovincia Mediterráneo-Iberolevantina, Rivas Martínez, 1987).

\section{Metodología}

Las asociaciones descritas para el territorio andaluz han sido estudiadas mediante la metodología fitosociológica (BraunBlanquet, 1979). Para cada una de ellas se estudió su posición sintaxonómica (Barkman etal., 1986), publicación original, fisiognomía, sinecología (Rivas Martínez, 1987), sincorología (Nieto et al., 1991; Rivas Martínez

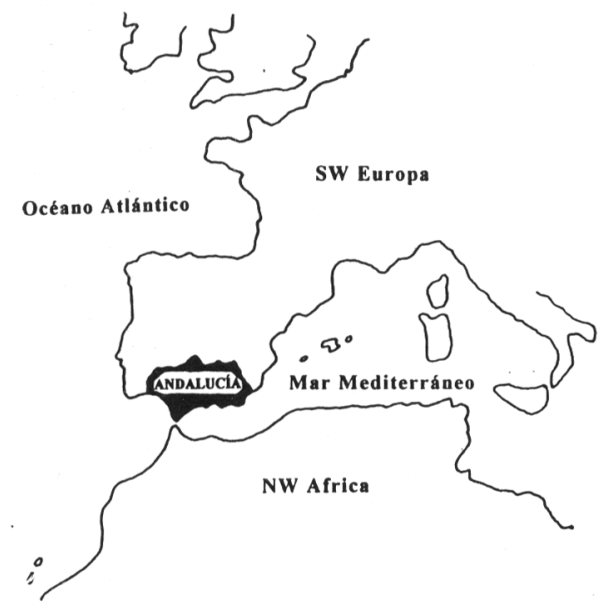

Figura 1. Situación del área de estudio (Andalucía, España; extensión $=87000 \mathrm{Km}^{2}$, temperatura media anual $=16,8^{\circ} \mathrm{C}$, precipitación media anual $=630 \mathrm{~mm}$.). Place of the stucy area (Andalusia, Spain, extension $=87.000 \mathrm{Km} 2$, annualmean temperature= $16,8^{\circ} \mathrm{C}$, annual mean rainfall $=630 \mathrm{~mm}$ ) et al., 1987 y Pérez Latorre et al., 1993, 1996) y sinfitosociología (Rivas Martínez, 1976; Cabezudo et al., 1987; Rivas Martínez et al., 1987 y Pérez Latorre et al., 1994). La nomenclatura de los taxones sigue a Valdés et al. (1987). En el esquema sintaxonómico se incluye también la variabilidad (a nivel de subasociación) de cada una de las asociaciones estudiadas.

\section{RESULTADOS}

Resumiendo los datos aportados por todos los autores previamente citados, podemos decir que los jarales sobre sustratos duros del orden Lavanduletalia stoechadis están representados en Andalucía por cuatro alianzas. Ulici-Cistion ladaniferi se distribuye por Andalucía Occidental y Calicotomo-Cistion ladaniferi lo hace por Andalucía Oriental. La alianza Stahelino-Ulicion baetici es endémica y se desarrolla sobre sustratos ígneos (peridotitas). En las altas montañas silíceas de Andalucía Oriental se encuentran las asociaciones de la alianza Cistion laurifolii. Los jaguarzales sobre sustratos arenosos del orden StauracanthoHalimietalia commutati están representados en Andalucía Occidental por la alianza Coremion albi que se distribuye tanto por arenales litorales como de interior.

Es destacable la gran diversidad de sintáxones existentes en el territorio andaluz (tab. 2), lo que se corresponde con una paralela riqueza en ambientes requeridos por estas formaciones. Sin embargo, se hace necesario un esfuerzo sintético sobre todo en aquellas asociaciones que ocupan areales muy puntuales, a las que correspondería el rango de subasociación.

Las asociaciones descritas para Andalucía (tab. 1) se ordenan según su status sintaxonómico y para cada una de ellas se cita el nombre, autor(es), publicación original, fisionomía, sinecología, sincorología (fig. 2), 


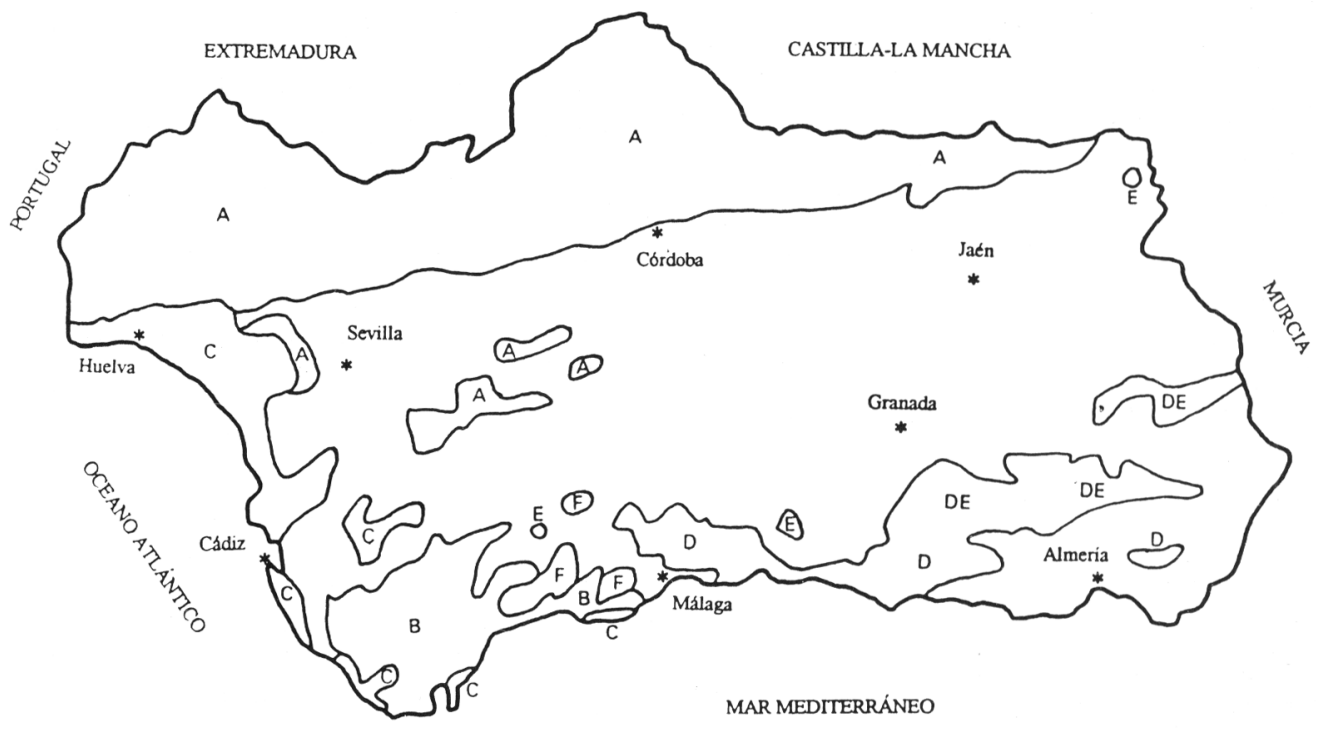

Figura 2. Distribución potencial en Andalucía de las alianzas y asociaciones estudiadas. A=Ulici argenteiCistion ladaniferi (subal. Ulici-Cistenion), B=Ulici argentei-Cistion ladaniferi (subal. Ulici-Calicotomenion villosae), $\mathrm{C}=$ Coremion albi, $\mathrm{D}=$ Calicotomo spinosae-Cistion ladaniferi (subal. Genistenion umbellatate), $\mathrm{E}=$ Cistion laurifolii, F=Stahelino-Ulicion baetici. Potential distribution in Andalusia of the studied alliances and associations .

sinfitosociología, especies características y/o diferenciales y comentarios sintaxonómicos donde fuese necesario.

En el esquema sintaxonómico final aparecen únicamente las asociaciones y subasociaciones que reconocemos para el territorio andaluz.

Cl. Cisto-Lavanduletea Br.-Bl. 1940 em. Rivas Martínez 1968. Matorrales silicícolas heliófilos y xerofíticos, sobre suelos meso-oligótrofos inmaduros o erosionados, de textura cohesiva y relativamente ricos en nutrientes (Lavanduletalia stoechidis) o de textura suelta y pobres en nutrientes (Stauracantho genistoidis-Halimietalia commutati). Región Mediterránea.

+ Lavanduletalia stoechadis Br-Bl. (1931) 1940 em. Rivas Martínez 1968. Comunidades de la subregión Mediterránea Occidental sobre sustratos duros.

\# Ulici argentei-Cistion ladaniferi $\mathrm{Br}$ - $-\mathrm{Bl}$., $\mathrm{P}$.
Silva \& Rozeira 1964 em. Rivas Martínez 1979. Asociaciones termo-mesomediterráneas iberomarroquí-atlánticas.

\#\# Ulici argentei-Cistenion ladaniferi. Asociaciones del SW de la Península Ibérica. Especies características: Ulex eriocladus, Lavandula luisieri, Cistus populifolius subsp. populifolius, Genista hirsuta subsp. hirsuta, Genista polyanthos, etc.

\section{Genisto hirsutae-Cistetum ladaniferi Rivas} Goday 1954 em. Rivas Martínez 1979

[Aportaciones a la fitosociología hispánica. Anales del Inst. Bot. Cav. 13:335-422. 1954]

Jaral meso-termomediterráneo secosubhúmedo del sector Mariánico, presente en las series de los alcornocales del SanguisorboQuerceto suberis $\mathrm{S}$, encinares del PyroQuerceto rotundifoliae $\mathrm{S}$ y encinares del Smilaci-Querceto rotundifoliae quercetoso suberis $\mathrm{S}$, caracterizado por Genista hirsuta y Astragalus lusitanicus. 
La importante presencia y cobertura de especies de Ulici-Cistion ladaniferi (Cistus ladanifer, Halimium umbellatum, Genista hirsuta subsp. hirsuta) y la posición ecológica intermedia entre las rañas de Sierra Morena y los arenales onubenses (Rivas Martínez, 1980) nos llevan a incluir en esta asociación al Halimio commutati-Cistetum libanotis Rivas Goday 1955 ex Rivas Martínez 1979 [Aportaciones a la fitosociología hispánica. Anales del Inst. Bot. Cav. 13:335-422. 1954] como subasociación psammófila (Genisto hirsutae-Cistetum ladaniferi cistetosum libanotis comb. et stat. nov.). Se trata de un jaguarzal-jaral termomediterráneo seco, que se desarrolla sobre arenas y rañas del límite entre los sectores Onubense y AracenoPacense, alcanzando puntualmente el Hispalense, presente en la serie de los alcornocales del Myrto-Querceto suberis halimietoso $S$ y caracterizado por Cistus libanotis y Halimium halimifolium.

La asociación Scillo-Lavanduletum pedunculatae Ladero 1970 [Contribución al estudio de la flora y vegetación de las comarcas de la Jara, Serranía de Ibor y GuadalupeViluercas en la Oretana central. Tesis Doctoral. Facultad de Farmacia. Madrid. 1970] es un cantuesal meso-termomediterráneo secosubhúmedo de la provincia LusoExtremadurense, citado en Andalucía en la serie de los encinares del Pyro-Querceto rotundifoliae $S$ y caracterizado por Scilla maritima y Lavandula sampaiana. Al menos en Andalucía, las formaciones semejantes a lo descrito en esta asociación parecen ser etapas intermedias entre el pastizal anual y el jaral de Genisto-Cistetum ladaniferi, debido a la capacidad primocolonizadora de las especies características de la comunidad.

\section{Erico australis-Cistetum populifolii Rivas Goday 1964 \\ [Vegetación y Flórula de la Cuenca Extremeña del Guadiana. Badajoz. 1964]}

Jaral-brezal mesomediterráneo subhúmedohúmedo del subsector Araceno-Pacense, presente en la serie de los alcornocales del Sanguisorbo-Querceto suberis $\mathrm{S}$ y caracterizado por Erica australis y Cistus populifolius subsp. populifolius.

\section{Ulici eriocladi-Cistetum ladaniferi Rivas Martínez 1979}

[Brezales y jarales de Europa Occidental. Lazaroa 1:5-128. 1979]

Jaral con aulagas meso-termomediterráneo subhúmedo del subsector Araceno-Pacense, presente en las series de los alcornocales del Sanguisorbo-Querceto suberis S y MyrtoQuerceto suberis lavanduletoso luisieri S y caracterizado por Ulex eriocladus y Lavandula luisieri.

La asociación Phlomido purpureaeCistetum albidi Rivas Martínez, Lousa, Díaz, Fernández González y Costa 1990 [La vegetación del sur de Portugal (Sado, Alentejo y Algarve). Itinera Geobotanica, 3:5-126. 1990] ha sido citada para Andalucía como matorral calcícola termo-mesomediterráneo subhúmedo del sector Algarviense y del subsector AracenoPacense, presente en las series de los encinares del Pyro-Querceto rotundifoliae $S$ y SmilacoQuerceto rotundifoliae $S$ y caracterizado por Cistus albidus y Phlomis purpurea. Debido a su composición florística y participación en series calcícolas, generalmente encinares de Pyro-Querceto rotundifoliae $S$ en su faciación basófila (Ladero et al., 1990) creemos que debe incluirse en Eryngio-Ulicion erinacei (Rosmarinetea officinalis). Los demás jarales con Cistus albidus y otros elementos calcícolas del subsector Araceno-Pacense, en su parte andaluza, sobre calizas descarbonatadas, de la serie de los alcornocales del SanguisorboQuerceto suberis $S$ creemos que podrían incluirse en Ulici eriocladi-Cistetum ladaniferi cistetosum albidi (Pérez Latorre et al., 1994). 
$n^{0}$ de orden

\section{$\begin{array}{llllllllllllllllllll}\text { A } & \text { B } & C & \text { D } & \text { E } & \text { F } & \text { G } & \text { H } & \text { I } & \text { J } & \text { K } & \text { L } & \text { M } & \text { N } & \text { O } & \text { P } & \text { Q } & \text { R } & \text { S }\end{array}$}

\section{Cisto-Lavanduletea, Lavanduletalia}

Cistus crispus

Cistus salvifolius

Cistus monspeliensis

Heichrysum serotinum

Lavandula stoechas

Halimium umbellatum

\section{Ulici argentei-Cistion ladaniferi}

Cistus ladanifer

\section{Ulici-Cistenion}

Genista hirsuta subsp. hirsuta

Lavandula sampaiana

Lavandula luisieri

Ulex eriocladus

Astragalus lusitanicus

Halimium ocymoides

Erica australis

Cistus populifolius subsp. populifolius

Cytisus bourgaei

Teucrium oxylepis

Centaurea citricolor

Ulici-Calicotomenion

Calicotome villosa

Ulex borgiae

Cistus populifolius subsp. major

Genista hirsuta subsp. lanuginosa

\section{Calicotomo spinosae-Cistion ladaniferi}

\section{Genistenion umbellatae}

Genista umbellata

Ulex parviflorus

Ptilostemon hispanicus

Teucrium haenseleri

Halimium atriplicifolium subsp. atriplicif.

Teucrium compactum

Festuca scariosa

\section{Cistion laurifolii}

Cistus laurifolius

Cytisus reverchonii

Dorycnium pentaphyllum

Catananche caerulea

Thymus gadorensis

Erinacea anthyllis

Centaurea sagredoi

\begin{tabular}{|c|c|c|c|c|c|c|c|c|c|c|}
\hline $\mathrm{V} \mathrm{V}$ IV & $\mathrm{p}$ & & II & . & & & IV & & & III \\
\hline IV $\mathrm{V} \quad \mathrm{V}$ & $\mathrm{p}$ & IV & II & + & p & p & IV & II & & V \\
\hline III . & $\mathrm{p}$ & . & III & II & - & . & . & . & . & . \\
\hline III III III & . & . & . & IV & . & $\mathrm{p}$ & & II & I & IV \\
\hline . $\mathrm{V}$ III & $\mathrm{p}$ & . & V & V & $\mathrm{p}$ & p & & II & - & V \\
\hline III V & $\mathrm{p}$ & IV & . & . & • & . & & V & • & . \\
\hline
\end{tabular}

$\begin{array}{lllllll}\mathrm{V} & \mathrm{V} & \mathrm{V} & \mathrm{p} & \mathrm{V} & \mathrm{III} \\ +\end{array}$

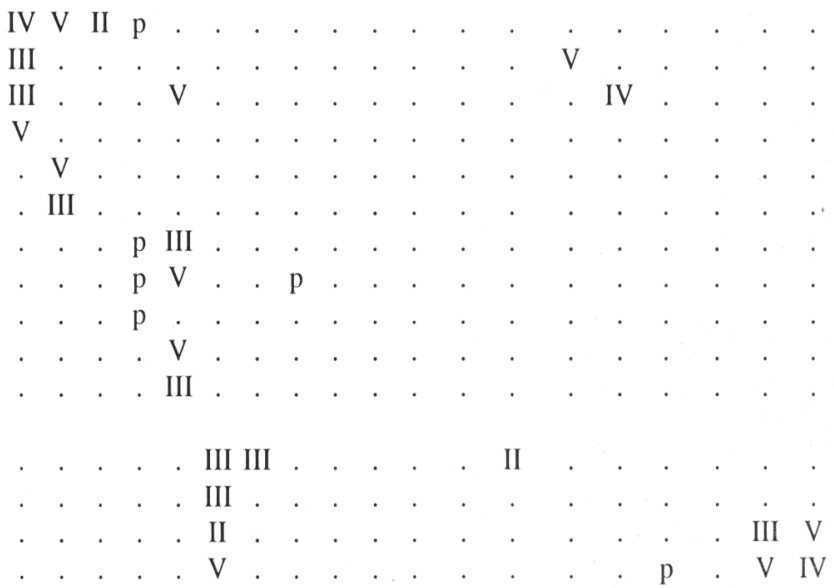

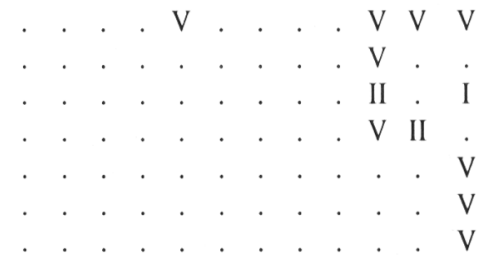




\begin{tabular}{|c|c|c|c|c|c|c|c|c|c|c|c|c|c|c|c|c|c|c|c|}
\hline$n^{0}$ de orden & A & B & $\mathrm{C}$ & D & $\mathbf{E}$ & $\mathbf{F}$ & G & $\mathbf{H}$ & I & $\mathbf{J}$ & $\mathbf{K}$ & $\mathbf{L}$ & $\mathbf{M}$ & $\mathbf{N}$ & $\mathbf{O}$ & $\mathbf{P}$ & $\mathbf{Q}$ & $\mathbf{R}$ & $\mathbf{S}$ \\
\hline Halimium halimifolium & . & . & $\mathrm{V}$ & . & . & . & . & . & . & . & . & . & V & V & II & . & . & . & . \\
\hline Halimium commutatum & . & . & $\mathrm{V}$ & . & . & . & . & . & . & . & . & . & IV & V & I & . & . & . & . \\
\hline Corema album & . & . & . & . & . & . & . & . & . & . & . & . & . & + & . & . & . & . & . \\
\hline Iberis welwistchii & . & . & . & . & . & . & . & . & . & . & . & . & + & I & . & . & . & . & . \\
\hline Stauracanthus genistoides & . & . & . & . & . & . & . & . & . & . & . & . & II & V & . & . & . & . & . \\
\hline Ulex australis & . & . & . & . & . & . & . & . & . & . & . & . & V & I & . & . & . & . & . \\
\hline Pterocephalus intermedius & . & . & . & . & . & . & . & . & . & . & . & . & + & . & . & . & . & . & . \\
\hline Armeria velutina & . & . & . & . & . & . & . & . & . & . & . & . & . & V & . & . & . & . & . \\
\hline Thymus tomentosus & . & . & . & . & . & . & . & . & . & . & . & . & . & V & . & . & . & . & . \\
\hline Dianthus broteri & . & . & . & . & . & . & . & . & . & . & . & . & . & III & . & . & . & . & . \\
\hline Fumana juniperina & . & . & . & . & . & . & . & . & . & . & . & . & . & . & V & . & . & . & . \\
\hline Eryngium dilatatum & . & . & . & . & . & . & . & . & . & . & . & . & . & . & III & . & . & . & . \\
\hline Klasea monardii & . & . & . & . & . & . & . & . & . & . & . & . & . & . & III & . & - & . & . \\
\hline Festuca caerulescens & . & . & . & . & . & . & . & . & . & . & . & . & . & . & II & . & . & . & . \\
\hline \multicolumn{20}{|l|}{ Stahelino-Ulicion baetici } \\
\hline Stahelina baetica & . & . & . & . & . & . & . & . & . & . & . & . & . & . & . & $\mathrm{p}$ & IV & V & II \\
\hline Ulex baeticus & . & . & . & . & . & . & . & . & . & . & . & . & . & . & . & $\mathrm{p}$ & IV & V & V \\
\hline Scorzonera baetica & . & . & . & . & . & . & . & . & . & . & . & . & . & . & . & $\mathrm{p}$ & IV & . & V \\
\hline Galium boissieranum & . & . & . & . & . & . & . & . & . & . & . & . & . & . & . & $\mathrm{p}$ & V & V & II \\
\hline Halimium atriplicif. subsp. serpentinicola & . & . & . & . & . & . & . & . & . & . & . & . & . & . & . & $\mathrm{p}$ & . & V & V \\
\hline Genista triacanthos & . & . & . & . & . & + & . & . & . & . & . & . & . & . & . & . & $\cdot$ & IV & III \\
\hline Serratula baetica & . & . & . & . & . & . & . & . & . & . & . & . & . & . & . & $\mathrm{p}$ & IV & V & $\cdot$ \\
\hline Linum sufruticosum subsp. carratracensis & . & . & . & . & . & . & . & . & . & . & . & . & . & . & . & $\mathrm{p}$ & V & III & . \\
\hline Digitalis laciniata & . & . & . & . & . & . & . & . & . & . & . & . & . & . & . & $\mathrm{p}$ & . & $\cdot$ & . \\
\hline Armeria colorata & . & . & . & . & . & . & . & . & . & . & . & . & . & . & . & . & . & IV & . \\
\hline Centaurea carratracensis & . & . & . & . & . & . & . & . & . & . & . & . & . & . & . & . & V & $\cdot$ & . \\
\hline Teucrium reverchonii & . & . & . & . & . & . & . & . & . & . & . & . & . & . & $\cdot$ & $\mathrm{p}$ & IV & . & . \\
\hline
\end{tabular}

Tabla 1. Tabla sintética de las asociaciones descritas para la clase Cisto-Lavanduletea en Andalucía (España). Se tienen en cuenta táxones característicos de unidades superiores, asociación y subasociáción. Synthetic table of the associations described for the class Cisto-Lavanduletea in Andalusia (Spain). Characteristic taxa of superior units, association and subassociation are taken into account.

Procedencia de los inventarios: A=Ulici eriocladi-Cistetum ladaniferi (Rivas Martínez, 1979), $\mathrm{B}=$ Genisto hirsutae-Cistetum ladaniferi (Rivas Goday, 1954), C=Halimio commutati-Cistetum libanotis (Rivas Goday, 1954),D=Erico australis-Cistetum populifolii (Rivas Goday, 1964), E=Teucrio marianiCistetum laurifolii (Velasco, 1980), $\mathrm{F}=$ Calicotomo villosae-Genistetum lanuginosae (Martínez Parras et al., 1987), G=Lavandulo caesiae-Genistetum equisetiformis (Rivas Goday y Rivas Martínez, 1967), $\mathrm{H}=$ Halimio atriplicifolii-Cistetum populifolii (Martínez Parras et al., 1987), I=Teucrio compacti-Cistetum ladaniferi (Martínez Parras et al., 1992), J=Cytiso reverchonii-Cistetum laurifolii (Valle et al., 1988), $\mathrm{K}=$ Halimio viscosii-Cistetum laurifolii (Martínez Parras y Molero Mesa, 1983), L=Thymo gadorensisCistetum laurifolii (Martínez Parras et al., 1987), $\mathrm{M}=$ Cisto salvifolii-Ulicetum australis (Pérez Latorre et al., 1993), $\mathrm{N}=$ Halimio halimifolii-Stauracanthetum genistoidis (Rivas Martínez et al., 1980), O=Fumano juniperinae-Cistetum crispi (Sánchez García y Galán de Mera, 1996), P=Halimio serpentinicolae-Digitaletum laciniatae (Rivas Goday y Rivas Martínez, 1967), Q=Asperulo hirsutae-Stahelinetum baeticae (Rivas Goday y Rivas Martínez, 1967), R=Armerio coloratae-Ulicetum baetici (Asensi y Díez, 1988), S=Genisto lanuginosae-Cistetum populifolii (Asensi y Díez, 1988). 


\section{Teucrio mariani-Cistetum laurifolii Velasco 1980}

[Comportamiento ecológico y fitosociológico de Teucrium oxylepis Font Quer subsp. marianum Ruiz de La Torre y Ruiz del Castillo. Anales Jard. Bot. Madrid 37:721-724. 1980]

[lectotypus designado aquí]

Jaral mesomediterráneo subhúmedo del subsector Marianense, presente en la serie de los alcornocales del Sanguisorbo-Querceto suberis $\mathrm{S}$ y caracterizado por Teucrium oxylepis subsp. marianum y Centaurea citricolor.

Asociación de transición al Cistion laurifolii con presencia muy puntual en Andalucía, en la zona de Despeñaperros (Aldeaquemada, Jaén). La presencia de $C$. laurifolius en estas localidades se entiende como debida a la extremada continentalidad y no por la altitud como en otras asociaciones andaluzas del Cistion laurifolii. La inexistencia de sintypus en la descripción original nos lleva a designar como lectosintypus al inventario $n^{\circ}$ 1 de la tabla correspondiente de dicha publicación (Velasco, 1980).

\section{\#\# Ulici borgiae-Calicotomenion villosae suball. nova}

Sintypus: Calicotomo villosae-Genistetum lanuginosae Martínez Parras, Peinado y De la Cruz 1987 corr. Pérez Latorre, Galán de Mera, Deil y Cabezudo 1996

Subalianza que engloba los jarales con aulagas propios del sector Aljíbico y que podría alcanzar el sector Tingitano, donde aparecen varias de las especies características. Se desarrolla sobre suelos silíceos degradados, bajo ombroclima subhúmedo-húmedo y como etapa de sustitución de alcornocales, quejigares y robledales.

Especies características: Calicotome villosa, Genista hirsuta subsp. lanuginosa, Ulex borgiae, Ulex baeticus subsp. baeticus (dif.), Cistus populifolius subsp. major.

\section{Calicotomo villosae-Genistetum} lanuginosae Martínez Parras, Peinado y De la Cruz 1987 corr. Pérez Latorre, Galán de Mera, Deil y Cabezudo 1996

[Aportación al estudio fitosociológico de los matorrales del sector Rondeño. Studia Botanica 6:39-45. 1987]

Jaral-aulagar termomediteráneo subhúmedohúmedo del sector Aljíbico, presente en las series de los alcornocales del Myrto-Querceto suberis $\mathrm{S}$ y Teucrio-Querceto suberis $\mathrm{S}$ y quejigares del Rusco-Querceto canariensis S, caracterizado por Calicotome villosa, Genista hirsuta subsp. lanuginosa y Ulex borgiae.

La asociación Ulici borgiae-Cistetum ladaniferi Asensi y Díez 1988 [Ulici borgiaeCistetum ladaniferi nueva asociación para el sector Gaditano (SW España). Homenaje a P. Monserrat: 405-407. Jaca-Huesca. 1988], descrita como jaral-aulagar termomediterráneo subhúmedo del sector Aljíbico, presente en las series de los alcornocales del Myrto-Querceto suberis $S$ y Teucrio-Querceto suberis $S$ y caracterizado por Ulex borgiae, fué incluída (p. p.) en el Calicotomo-Genistetum lanuginosae por Pérez Latorre et al. (1993).

\# Calicotomo-Cistion ladaniferi $\mathrm{Br}$--B1. (1931) 1940 em. Rivas Martínez 1979. Asociaciones meso-termomediterráneas, íbero-levantinas, languedocino-provenzales y béticas orientales. \#\# Genistenion umbellatae Peinado, Alcaraz y Martínez Parras 1992. Asociaciones béticas orientales y almerienses occidentales.

Especies características y/o diferenciales: Genista umbellata subsp. equisetiformis, Calicotome intermedia, Ulex parviflorus, Ptilostemon hispanicus, Halimium atriplicifolium, etc.

6. Lavandulo caesiae-Genistetum equisetiformis Rivas Goday y Rivas Martínez 1968

[Matorrales y tomillares de la península Ibérica comprendidos en la clase Ononido-Rosmarinetea. 
An. Inst. Bot. Cav. 25:5-180. 1967]

Jaral con bolinas termo-mesosupramediterráneo seco-subhúmedo de los subsectores Malacitano, Axarquiense y Almijarense, presente en las series de los encinares del Smilaci-Querceto rotundifoliae $S$, alcornocales con encinas del TeucrioQuerceto suberis quercetoso rotundifoliae $S$, encinares del Adenocarpo-Querceto rotundifoliae $S$ y robledales del AdenocarpoQuerceto pyrenaicae $S$, caracterizado por Genista umbellata y Ulex parviflorus.

Una variante de este jaral, puesta de manifiesto por Pérez Latorre et al. (1994), que se desarrolla sobre micaesquistos y cuarcitas en el subsector Axarquiense oriental y en el Malacitano litoral (sector MalacitanoAxarquiense, provincia Bética sensu Nieto et al., 1991), bajo ombroclima seco inferior, con Calicotome intermedia, nos lleva a describir la subasociación calicotometosum intermediae subass. nova; sintypus: MALAGA, Torrox, Cerro Gordo, $50 \mathrm{~m}^{2}$, altitud: $350 \mathrm{~m}$, filitas y cuarcitas. Características de asociación y subasociación: Calicotome intermedia 2, Cistus monspeliensis 4, Lavandula stoechas + , Ulex parviflorus 1, Adenocarpus telonensis +, Genista umbellata +. Compañeras: Phlomis purpurea 1, Daphne gnidium + , Inula viscosa 1, Tuberaria guttata + , Arisarum vulgare + , Asparagus acutifolius,+ Carthamus arborescens + , Selaginella denticulata + .

Dicha subasociación aparece como etapa de degradación de los encinares silicícolas con alcornoques de Smilaci-Querceto rotundifoliae quercetoso suberis $S$.

7. Teucrio compacti-Cistetum ladaniferi Martínez Parras, Peinado y Alcaraz in Peinado, Alcaraz y Martínez Parras 1992

[Vegetation of Southeatern Spain. Flora et vegetatio mundi. Band X. J. Cramer. 1992]

Jaral con lastones mesomediterráneo seco de los subsectores Nevadense, Alpujarreño, Serrano-Estanciense y Almeriense, presente en la serie de los encinares del AdenocarpoQuerceto rotundifoliae $S$ y caracterizado por Teucrium compactum y Festuca scariosa.

La localización puntual en la parte superior de la Sierra de la Contraviesa (Granada) del Halimio atriplicifolii-Cistetum populifolii Martínez Parras, Peinado y Alcaraz 1987 [Comunidades Vegetales de Sierra Nevada (España) 1987] y la distribución más amplia de Teucrio-Cistetum ladaniferi, llevan a incluir dentro de ésta a la primera como subasociación ombrófila (Teucrio compactiCistetum ladaniferi cistetosum populifolii comb. et stat. nov.). Constituye un jaral con jaguarzos mesomediterráneo subhúmedo del subsector Alpujarreño, presente en la serie de los encinares con alcornoques del AdenocarpoQuerceto rotundifoliae quercetoso suberis $S$ y caracterizado por Halimium atriplicifolium y Cistus populifolius subsp. populifolius.

\# Cistion laurifolii Rivas Goday in Rivas Goday et al. 1956 em. Rivas Martínez 1979. Asociaciones de óptimo supramediterráneo y mesomediterráneo superior distribuidos fundamentalmente por la provincia CarpetanoIbérico-Leonesa con irradiaciones a 10 mariánico y bético.

Especies características y/o diferenciales: Cistus laurifolius, Dorycnium pentaphyllum.

\section{Halimio viscosii-Cistetum laurifolii} Martínez Parras y Molero Mesa 1983

[Los melojares béticos y sus etapas de sustitución. Lazaroa 4:91-104. 1983]

Jaral con jaguarzos supramediterráneo subhúmedo del subsector Nevadense occidental y Almijaro-Granatense, presente en las series de los encinares del Adenocarpo-Querceto rotundifoliae $S$ y robledales del AdenocarpoQuerceto pyrenaicae $S$ y caracterizado por Halimium umbellatum subsp. viscosum y Cistus salvifolius. 


\section{Thymo gadorensis-Cistetum laurifolii} Martínez Parras, Peinado y Alcaraz 1987 [Datos sobre la vegetación de Sierra Nevada. Lazaroa 7:515-533. 1987]

Jaral con tomillos supramediterráneo seco de los subsectores Filábrico y Nevadense oriental, presente en la serie de los encinares del Adenocarpo-Querceto rotundifoliae $S$ y caracterizado por Thymus serpylloides subsp. gadorensis, Erinacea anthyllis y Centaurea sagredoi.

\section{Cytiso reverchonii-Cistetum laurifolii} Valle, Gómez Mercado y Mota 1988

[Los robledales de la Sierra de Segura y otras comunidades relacionadas con ellos. Anales Jard. Bot. Madrid 45(1):247-257.1988]

Jaral con escobones supramediterráneo subhúmedo-húmedo del sector Subbético, presente en la serie de los robledales del Berberido-Querceto pyrenaicae $S$ y caracterizado por Cytisus reverchonii y Dorycnium pentaphyllum.

A pesar de su área puntual, debida a limitaciones litológicas y no climáticas, su originalidad como integrante de la serie alcaracense del roble melojo, y su aislamiento respecto a asociaciones próximas confirman su status de asociación.

\section{Comunidad de Cistus laurifolius y Erica australis}

Dentro de esta alianza se ha puesto de manifiesto (Pérez Latorre, 1993) la existencia de una comunidad de Cistus laurifolius y Erica australis. Es un jaral con brezos mesomediterráneo húmedo, sobre brechas dolomíticas y micaesquistos del sector Rondeño. Está presente en la serie de los encinares con alcornoques del PaeonioQuerceto rotundifoliae quercetoso fagineae $S$ variante de Quercus suber, y a veces en los pinsapares de Paeonio-Abieteto pinsapo S, caracterizándose por Ulex baeticus y Erica australis.
\# Stahelino baeticae-Ulicion baetici Rivas Goday y Rivas Martínez 1969. Asociaciones serpentinícolas bermejense-carratracenses (provincia Bética).

Especies características: Stahelina baetica, Cistus ladanifer subsp. africanus, Galium boissieranum, Digitalis laciniata, Centaurea carratracensis, Teucrium reverchonii, Ulex baeticus, etc.

\section{Halimio serpentinicolae-Digitaletum} laciniatae Rivas Goday y Rivas Martínez 1967 [Matorrales y tomillares de la península Ibérica comprendidos en la clase OnonidoRosmarinetea. An. Inst. Bot. Cav. 25:5-180. 1967]

Jaguarzal meso-supramediterráneo subhúmedo-húmedo del sector Bermejense, presente en las series de los pinares del Pino pinastri-Querceto cocciferae $S$ y pinsapares del Bunio macucae-Abieteto pinsapo $S$, caracterizado por Digitalis laciniata, Halimium atriplicifolium y Armeria colorata.

\section{Asperulo hirsutae-Stahelinetum baeticae} Rivas Goday y Rivas Martínez 1967

[Matorrales y tomillares de la península Ibérica comprendidos en la clase OnonidoRosmarinetea. An. Inst. Bot. Cav. 25:5-180. 1967]

Matorral termo-mesomediterráneo secosubhúmedo del sector Bermejense presente en la serie de los pinares del Pino-Querceto cocciferae $S$ y caracterizado por Galium boissieranum y Stahelina baetica.

\section{Genisto lanuginosae-Cistetum populifolii} Asensi y Díez 1988

[Matorrales y jarales del sector Rondeño. Documents Pthytosociologiques 11:269. 273.1988]

Jaral con brezos mesomediterráneo húmedo del sector Bermejense en la serie de los pinsapares del Bunio-Abieteto pinsapo S, caracterizado por Cistus populifolius subsp. 
major, Erica scoparia y Genista triacanthos. Asociación que comparte cierto fondo florístico con la subalianza Ulici borgiaeCalicotomenion villosae.

La asociación Armerio colorataeUlicetum baetici Asensi y Díez 1988 [Matorrales y jarales del sector Rondeño. Documents Pthytosociologiques 11:269273.1988], descrita como matorral mesomediterráneo húmedo del sector Bermejense que se desarrolla en las series de los pinares del Pino-Querceto cocciferae $S$ y pinsapares del Bunio-Abieteto pinsapo $S$, caracterizado por Armeria colorata, Ulex baeticus y Linum carratracensis, parece ser un sinónimo con posición intermedia entre Genisto-Cistetum populifolii y HalimioDigitaletum laciniatae en base a su composición florística y posición ecológica casi idénticas.

+ Stauracantho genistoidis-Halimietalia commutati Rivas Martínez, Lousa, Díaz, Fernández González y Costa 1990. Comunidades de la subregión Mediterránea Occidental sobre sustratos arenosos.

\# Coremion albi Rothmaler 1954 (Stauracantho genistoidis-Halimion halimifolii Rivas Martínez, 1979). Asociaciones TingitanoOnubo-Algarvienses.

Especies características: Cistus libanotis, Halimium halimifolium, Halimium commutatum, Corema album, Stauracanthus genistoides, Ulex australis, etc.

15. Ulici subsericei-Cistetum libanotis (Br.Bl., Silva y Rozeira 1964) Rivas Martínez 1979 nom. nov.

[Landes à Cistes et Ericacées (CistoLavanduletea et Calluno-Ulicetea). Agron. Lusit. 23(4):229-313. 1964]

Jaguarzal con aulagas termomediterráneo seco, sobre arenas del sector Algarviense, presente en la serie de los sabinares del OsyrioJunipereto turbinatae $S$ y caracterizado por
Ulex argenteus subsp. subsericeus y Armeria macrophylla.

Esta asociación parece alcanzar el ex tremo occidental del litoral onubense, ya que los táxones citados llegan a dicho territorio (Valdés et al., 1987).

\section{Halimio halimifolii-Stauracanthetum} genistoidis Rivas Martínez, Costa, Castroviejo y Valdés 1980

[Vegetación de Doñana (Huelva, España). Lazaroa 1(2):5-189. 1980]

Jaguarzal con aulagas termomediterráneo seco sobre arenas del sector Onubense, presente en las series de los sabinares del OsyrioJunipereto turbinatae $\mathrm{S}$ y alcornocales del Myrto-Querceto suberis halimietoso $\mathrm{S}$ y caracterizado por Armeria velutina, Thymus tomentosus y Helichrysum picardii.

17. Cisto salvifolii-Ulicetum australis Pérez Latorre, Nieto y Cabezudo 1993

[Contribución al conocimiento de la vegetación de Andalucía. II. Los alcornocales. Acta Bot. Malacitana 18: 223-258.'1993]

Jaguarzal con aulagas termomediterráneo subhúmedo, sobre arenas del sector Aljíbico, presente en la serie de los alcornocales del Myrto-Querceto suberis halimietoso $S$ y caracterizado por Ulex australis, Cistus crispus y Calicotome villosa.

\section{Fumano juniperinae-Cistetum crispi} Sánchez García y Galán de Mera 1996

[Sobre la posición fitosociológica de Fumana juniperina (Cistaceae) en el SW de la península Ibérica. Acta Bot. Malacitana 21: 322-325. 1996]

Jaguarzal-tomillar termomediterráneo seco, sobre conglomerados del sector Onubense litoral (occidente de la provincia de Cádiz), presente en la serie de los alcornocales del Myrto-Querceto suberis halimietoso S y 
caracterizado por Fumana juniperina y Klasea monardii.

\section{ESQUEMA SINTAXONÓMICO}

A modo de conclusión, hemos elaborado el siguiente esquema sintaxonómico sobre la clase fitosociológica Cisto-Lavanduletea en Andalucía (España).

CISTO-LAVANDULETEA Br.-Bl. $1940 \mathrm{em}$. Rivas Martínez 1968

+ Lavanduletalia stoechidis Br-Bl. (1931) 1940 em. Rivas Martínez 1968

\# Ulici argentei-Cistion ladaniferi Br.-Bl. P. Silva y Rozeira 1964 em. Rivas Martínez 1979 \#\# Ulici argentei-Cistenion ladaniferi

1. Genisto hirsutae-Cistetum ladaniferi Rivas Goday 1954 em. Rivas Martínez 1979

cistetosum ladaniferi

ericetosum australis Rivas Goday 1955

halimietosum viscosii Pérez Latorre, Nieto

y Cabezudo 1993

cistetosum libanotis (Rivas Goday 1954 ex Rivas Martínez 1979) comb. et stat. nov.

2. Erico australis-Cistetum populifolii Rivas

Goday 1964

cistetosum populifolii

halimietosum viscosii Pérez Latorre, Nieto y Cabezudo 1993

3. Ulici eriocladi-Cistetum ladaniferi Rivas Martínez 1979

cistetosum ladaniferi

ericetosum australis Pérez Latorre, Nieto y Cabezudo 1993

cistetosum albidi Pérez Latorre, Nieto y Cabezudo 1994

4. Teucrio mariani-Cistetum laurifolii Velasco 1980 lectotipificada

\#\# Ulici borgiae-Calicotomenion villosae suball. nova

5. Calicotomo villosae-Genistetum lanuginosae Martínez Parras, Peinado y De la Cruz 1987 corr. Pérez Latorre, Galán de Mera, Deil y

Cabezudo 1996

genistetosum lanuginosae

genistetosum umbellatae Martínez Parras,

Peinado y De la Cruz 1987

halimietosum serpentinicolae Asensi y Díez 1988

genistetosum triacanthi Pérez Latorre, Nieto y Cabezudo 1993

\# Calicotomo-Cistion ladaniferi Br.-B1. (1931)

1940 em. Rivas Martínez 1979

\#\# Genistenion umbellatae Peinado, Alcaraz y Martínez Parras 1992

6. Lavandulo caesiae-Genistetum equisetiformis Rivas Goday y Rivas Martínez 1968 ex Martínez Parras, Peinado y Alcaraz 1985 genistetosum equisetiformis cistetosum laurifolii Martínez Parras, Peinado y Alcaraz 1987

thymetosum capitati Martínez Parras, Peinado y Alcaraz 1987

calicotometosum intermediae subass. nova

7. Teucrio compacti-Cistetum ladaniferi Martínez Parras, Peinado y Alcaraz in Peinado, Alcaraz y Martínez Parras 1992 cistetosum ladaniferi

cistetosum populifolii (Martínez Parras, Peinado y Alcaraz 1987) comb. et stat nov. \# Cistion laurifolii Rivas Goday in Rivas Goday et al. 1956 em. Rivas Martínez 1979

8. Halimio viscosi-Cistetum laurifolii Martínez Parras y Molero Mesa 1983

9. Thymo gadorensis-Cistetum laurifolii Martínez Parras, Peinado y Alcaraz 1987

10. Cytiso reverchonii-Cistetum laurifolii Valle, Gómez-Mercado y Mota 1988

11. Comunidad de Cistus laurifolius y Erica australis

\# Stahelino baeticae-Ulicion baetici Rivas Goday y Rivas Martínez 1969

12. Halimio serpentinicolae-Digitaletum laciniatae Rivas Goday y Rivas Martínez 1967

13. Asperulo hirsutae-Stahelinetum baeticae 

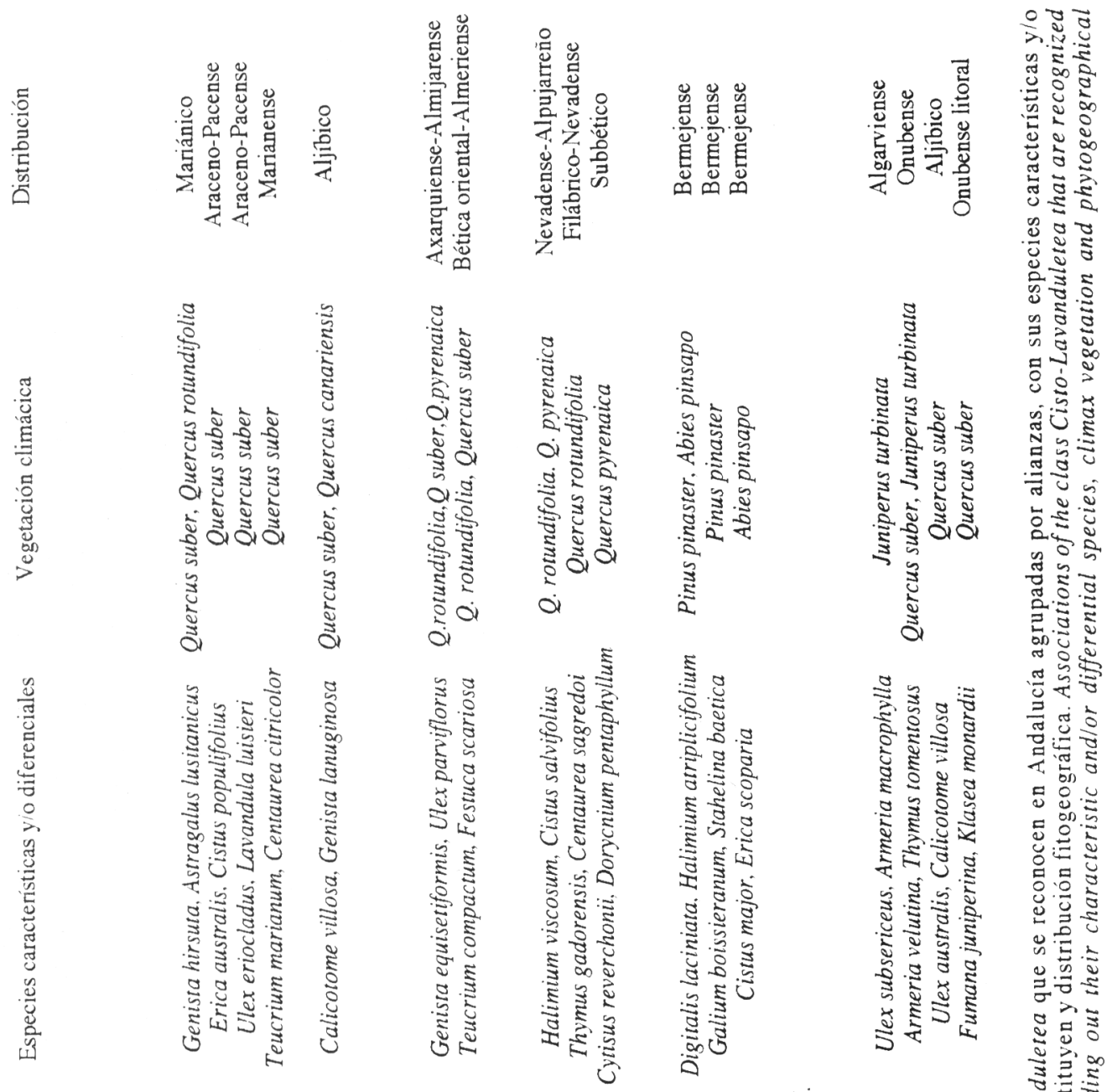

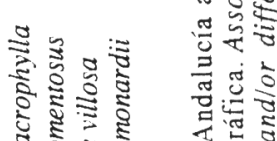
इ ₹

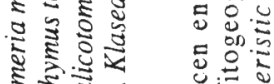

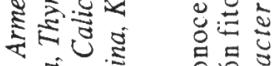

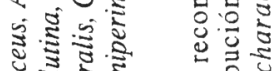

कू

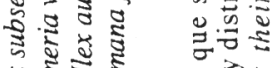

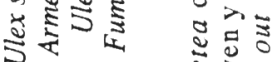

(2)

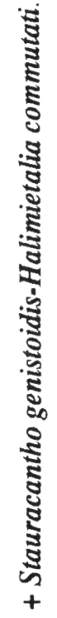

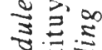

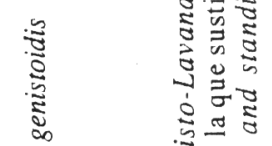

3 है

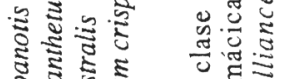

ปิ

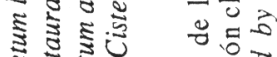

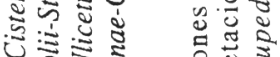

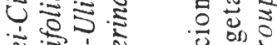

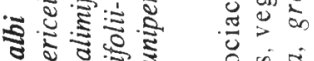

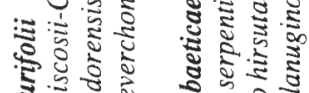

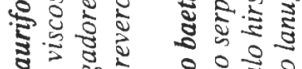

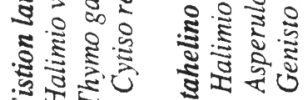

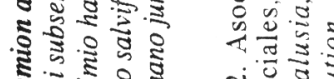

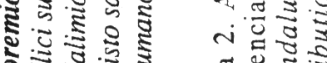

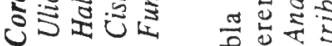

* 
Rivas Goday y Rivas Martínez 1967

14. Genisto lanuginosae-Cistetum populifolii Asensi y Díez 1988

+ Stauracantho genistoidis-Halimietalia commutati Rivas Martínez, Lousa, Díaz, Fernández González y Costa 1990

\# Coremion albi Rothmaler 1954

15. Ulici subsericei-Cistetum libanotis (Br.B1., Silva y Rozeira 1964) Rivas Martínez 1979 nom. nov.

16. Halimio halimifolii-Stauracanthetum genistoidis Rivas Martínez, Costa, Castroviejo y Valdés 1980

stauracanthetosum genistoidis

ulicetosum australis Rivas Martínez, Costa, Castroviejo y Valdés 1980

17. Cisto salvifolii-Ulicetum australis Pérez Latorre, Nieto y Cabezudo 1993

18. Fumano juniperinae-Cistetum crispi Sánchez García y Galán de Mera 1996

\section{BIBLIOGRAFÍA}

ACHHAL, A., O. AKABLI, M. BARBERO, A. BENABID, A. M'HIRIT, C. PEYRE, P. QUÉZEL \& S. RIVAS MARTÍNEZ -1979- A propos de la valeur bioclimatique et dynamique de quelques essences forestières au Maroc. Ecologia Mediterranea 5:211-249.

ASENSI, A. y B. DÍEZ GARRETAS-1987Andalucía Occidental. En: La vegetación de España. Peinado Lorca y S. Rivas Martínez (eds.).

ASENSI, A. y B. DÍEZ GARRETAS - 1988- Ulici borgiae-Cistetum ladaniferi, nueva asociación para el Sector Gaditano ( $S W$ de España). Homenaje a P. Montserrat: 405-407. Jaca y Huesca.

BARKMAN, J. J., J. MORAVEC \& S. RAUSCHERT -1986-Code of phytosociological nomenclature. Vegetatio 67(3): 145-195.

BENABID, A. -1984-Étude phytoecologique des pleupements forestiers et preforestiers du Rif centro-occidental (Maroc). Travaux de l'Institut Scientifique. Série Botanique 34. Rabat.

BENABID, A. \& M. FENNANE -1994Connaisances sur la végétation du Maroc:
Phytogéographie, phytosociologie et séries de végétation. Lazaroa 14:21-97.

BRAUN BLANQUET, A. PINTO DA SILVA \& A. ROZEIRA -1964- Landes à Cistes et Ericacées (Cisto-Lavanduletea et Calluno-Ulicetea). Agron. Lusit. 23(4):229-313.

BRAUN-BLANQUET, J., 1979. Fitosociología. Ed. Blume.

CABEZUDO, B., J.M. NIETO CALDERA y A.V. PÉREZ LATORRE -1989- Contribución al estudio edafófilo-serpentinícola del sector Rondeño (Málaga, España). Acta. Bot. Malacitana 14:291-294.

CANO, E., F. VALLE y E. ARROJO - 1991- Estudio fitosociológico de los matorrales del Parque Natural de Andújar (Jaén/España) Monogr. Fl. Veg. Beticas 6:55-82.

CANO, E., A. GARCÍA FUENTES y N. SÁNCHEZ PASCUAL - 1993-Vegetación de la Cordillera Mariánica y Cuenca del Guadiana. Dpto. Biol. Vegetal. Univ. Jaén.

CEBALLOS, L. y C. VICIOSO - 1933-Estudio sobre la vegetación y la flora forestal de la provincia de Málaga. IX Inspección del I.C.O.N.A. Madrid.

CEBALLOS, L. y M. MARTÍN BOLAÑOS -1930Estudio sobre la vegetación forestal de la provincia de Cádiz. Ingenieros de Montes del I.F.I.E. Madrid.

ESCUDERO, A., F. FERNÁNDEZ GONZÁLEZ, R. GAVILÁN y A. RUBIO -1995-Revisión nomenclatural de la alianza Cistion laurifolii Rivas Goday 1956 y sus sintáxones subordinados. Lazaroa, 16:172-181.

FERNÁNDEZ GALIANO, E. -1990- Pasado, presente y futuro de los bosques de la Península Ibérica. Acta Bot. Malacitana. 15:135-143.

LADERO, M. -1970- Contribución al estudio de la flora y vegetación de las comarcas de la Jara, Serranía de Ibor y Guadalupe-Viluercas en la Oretana central. Tesis Doctoral. Facultad de Farmacia. Madrid.

LADERO, M., J.L. PÉREZ CHISCANO, M.T. SANTOS, C.J. VALLE y A. AMOR -1990Encinares Luso-Extremadurenses y sus etapas preclimácicas. Acta Bot. Malacitana 15:323-329.

MARTÍNEZ PARRAS J.M. y J. MOLERO MESA 1982- Los melojares béticos y sus etapas de sustitución. Lazaroa 4:91-104.

MARTÍNEZ PARRAS, J.M., M. PEINADO y F. ALCARAZ -1986- Sobre la vegetación termófila de la cuenca mediterránea de Granada y sus 
áreas limítrofes. Lazaroa 8:251-268.

MARTÍNEZ PARRAS, J.M. y M. PEINADO LORCA -1987- Andalucía Oriental. En: La vegetación de España. Peinado Lorca, M. y S. Rivas Martínez (eds.).

MARTÍNEZ PARRAS, J.M., M. PEINADO y F. ALCARAZ -1987- Comunidades Vegetales de Sierra Nevada. Monografías de la Universidad de Alcalá de Henares 1:33-37.

MARTÍNEZ PARRAS, J.M., M. PEINADO y F. ALCARAZ - 1987- Datos sobre la vegetación de Sierra Nevada. Lazaroa 7:515-533.

MARTÍNEZ PARRAS, J.M., M. PEINADO y M. DE LA CRUZ -1987- Aportación al estudio fitosociológico de los matorrales del Sector Rondeño. Studia Botanica 6:39-45

MOLINA ABRIL, J.A. -1993-Resumen sintaxonómico de las comunidades vegetales de Francia y España hasta el rango de alianza. Colloques phytosociologiques 22:55-110.

NIETO CALDERA, J.M., A.V. PÉREZ LATORRE y B. CABEZUDO -1991-Biogeografía y series de vegetación de la provincia de Málaga (España). Act. Bot. Malacitana 16(2):417-436.

PEINADO, M., F. ALCARAZ \& J.M. MARTÍNEZ PARRAS -1992-Vegetation of Southeatern Spain. Flora et vegetatio mundi. Band X. J. Cramer.

PÉREZ LATORRE, A.V. -1993- Estudio Fitocenológico de los Alcornocales Andaluces. Tesis Doctoral. Universidad de Málaga.

PÉREZ LATORRE, A.V., J.M. NIETO y B CABEZUDO -1993-Contribución al conocimiento de la vegetación de Andalucía. II. Los alcornocales. Acta Bot. Malacitana 18: 223-258

PÉREZ LATORRE, A.V., J.M. NIETO CALDERA y B. CABEZUDO -1994- Datos sobre la vegetación de Andalucía III. Series de vegetación caracterizadas por Quercus suber $\mathrm{L}$. Acta Bot. Malacitana 19: 169-185.

PÉREZ LATORRE, A.V., A. GALÁN DE MERA, U. DEIL y B. CABEZUDO -1996- Fitogeografía y vegetación del sector Aljíbico (Cádiz-Málaga, España). Acta Bot. Malacitana 21: (241-267).

QUÉZEL, P., M. BARBERO, A. BENABID, M. LOISEL \& S. RIVAS MARTINEZ -1988Contribution a l'etude des groupements préforestiers et des matorrals rifains. Ecol. Med. 14(1): 77-122.

RIVAS GODAY, S. -1954- Aportaciones a la fitosociología hispánica. An. Inst. Bot. Cav. 13:335-423.
RIVAS GODAY, S. -1964a-Aportaciones a la fitosociología hispánica. An. Inst. Bot. Cav. 22:341-405.

RIVAS GODAY, S. -1964b- Vegetación y flórula de la cuenca extremeña del Guadiana. Serv. Publ. Excma. Dip. Prov. Badajoz.

RIVAS GODAY, S. y F. BELLOT -1944- Estudios sobre la vegetación y flora de la comarca de Despeñaperros-Santa Elena. An. Jard. Bot. 5:377-503.

RIVAS GODAY, S. y S. RIVAS MARTÍNEZ 1967-Matorrales y Tomillares de la Península Ibérica comprendidos en la clase OnonidoRosmarinetea. An. Inst. Bot. Cav. 25:5-180

RIVAS MARTÍNEZ, S. -1976- Sinfitosociología, una nueva metodología para el estudio del Paisaje Vegetal. An. Inst. Bot. Cav. 33:179-188.

RIVAS MARTÍNEZ, S. -1979- Brezales y jarales de Europa Occidental (Revisión de las clases Calluno-Ulicetea y Cisto-Lavanduletea). Lazaroa 1:5-128.

RIVAS MARTÍNEZ, S. -1987- Memoria y Mapas de las Series de Vegetación de España. M. A. P. A.-I. C. O. N. A.

RIVAS MARTÍNEZ, S., M. COSTA, S. CASTROVIEJO y B.VALDÉS -1980-Vegetación de Doñana. Lazaroa 2:5-189.

RIVAS MARTÍNEZ, S., M. LOUSA, T. E. DÍAZ, F. FERNÁNDEZ GONZALEZ y J. COSTA. -1990La vegetación del sur de Portugal (Sado, Alentejo y Algarve). Itinera Geobotanica 3: 5-126.

SÁNCHEZ GARCÍA, I. y A. GALÁN DE MERA 1996- Sobre la posición fitosociológica de Fumana juniperina (Cistaceae) en el SW de la península Ibérica. Acta Bot. Malacitana 21: (322-325).

VALDÉS, B., S. TALAVERA y E.F. GALIANO (eds.) -1987- Flora vascular de Andalucía Occidental. Ed. Ketres. Barcelona.

VALLE, F., F. GÓMEZ MERCADO y F.J. MOTA 1988- Los robledales de la Sierra de Segura y otras comunidades relacionadas con ellos. Anales Jard. Bot. Madrid 45(1):247-257.

VELASCO, A. -1980-Comportamiento ecológico y fitosociológico de Teucrium oxylepis Font Quer subsp. marianum Ruiz de La Torre y Ruiz del Castillo. Anales Jard. Bot. Madrid 37:721-724.

Aceptado para su publicación en Mayo 1997

Dirección de los autores: Departamento de Biología Vegetal. Universidad de Málaga. A partado 59, 29080 Málaga. España. E-mail: avperez@uma.es 